\title{
Evaluation of laminated shaly sand sequences in Ahwaz oil field using (via) high-resolution square logs
}

\author{
Saeed Parvizi Ghaleh ${ }^{1} \cdot$ Ali Kordavani ${ }^{2}$
}

Received: 28 August 2017 / Accepted: 6 May 2019 / Published online: 20 May 2019

(c) The Author(s) 2019

\begin{abstract}
Thinly bedded and laminated shale-sand sequences are very common in most of the producing formations in Iran. Historically, traditionally well logging methods such as conventional resistivity measurement is employed to evaluate pay intervals in order to extract information about hydrocarbon saturation. However, there is the practical matter of resolution of these methods to detect thin laminated shaly sand layers. In order to cope with this limitation, other technique such as: highresolution method should be utilized. For doing this method, we receive low-resolution logs and a high-resolution log from a well logging tool that is disposed in a wellbore which has laminated shaly sand layers. Firstly, square log is produced then these logs are convolved with vertical response function of well logging tools to generate constructed log. The constructed logs are compared with low-resolution logs, if these two logs are matched we choose that square logs as optimum square $\operatorname{logs}$ and analyze them. But if these two logs do not match, the square logs undergo interactive refinement then these refined logs are subsequently convolved with a vertical response function of the well logging tool thereby producing a constructed $\log$. Then these constructed logs compare with low-resolution logs. This work repeated until these logs are matched and the optimum square logs are chosen. High-resolution square log method is an iterative method, time consuming and also has very mathematical calculations because we developed a computer program with MATLAB software which can do all these works with high accuracy and low time.
\end{abstract}

Keywords Laminated shaly sand (LSS) Conventional log · Petrophysical analysis · High-resolution square log method · Convolution $\cdot$ MATLAB software

\begin{tabular}{|c|c|}
\hline \multicolumn{2}{|c|}{ Abbreviations } \\
\hline LSS & Laminated shaly sand \\
\hline LSSA & Laminated shaly sand analysis \\
\hline RHOB & $\begin{array}{l}\text { Compensated gamma-gamma density average } \\
{\left[\mathrm{g} / \mathrm{cm}^{3}\right]}\end{array}$ \\
\hline CGR & Compensated gamma-ray [API] \\
\hline NPHI & Neutron porosity hydrogen index \\
\hline GUI & Graphical user interface \\
\hline NISOC & National Iranian South Oil Company \\
\hline
\end{tabular}

Electronic supplementary material The online version of this article (https://doi.org/10.1007/s13202-019-0674-1) contains supplementary material, which is available to authorized users.

Saeed Parvizi Ghaleh

Saeed.parvizi68@gmail.com

1 Department of Petroleum Engineering, Sahand University of Technology, Tabriz, Iran

2 Department of Petrophysics Engineering, National Iranian South Oil Company, Ahwaz, Iran

\section{Introduction}

Increased resolution from well logs is becoming a vital part of hydrocarbons explorations. New logging tools are being built with the particular focus of improving resolution to detect thin-bed pay. Shaly sand intervals in the Asmari Formation of the Ahwaz fields have been recently reported by National Iranian Oil Company (NIOC). These intervals include laminated sand-shale sequences, where dense sand layers exist within the shales. These thin-bed pays have been eluding the well logging methods (i.e., conventional resistivity measurements) for reserves estimates. The conventional resistivity measurements are generally influenced by the conductive formation. In the laminated shaly sand sequences, the shaly part of these layers is more conductive than the sandy part which in turn yields incorrect estimation of the hydrocarbon in laminated shaly sand layers. (Azim et al. 2005). Inherently, application of these methods generally underestimated hydrocarbon in place in these layers. To tackle this problem, new high technology logging 
measurement (such as: 3DEX multi-component induction tool) is required. These advanced logging measurements can efficiently measure the amount of the resistivity of each layer and quantify the existence of hydrocarbon bearing sand layers. (Zhang 2007). But these high technology logging measurements are too expensive that could render today's usage inapplicable.

There are different ways for analyzing of this layers better and enhancing the production from these reservoirs, which can be used for identifying the layers clearly and calculating petrophysical properties. The methods for investigation of laminated shaly sands are:

- Coring (Gabriela and Lorne 2000)

- NMR log (Coates et al. 1999)

- Resistivity anisotropy tools (Vielma and Vandini 2007)

- Image log and analysis of high-resolution square logs (Tabanou and Antoine 1995)

Therefore, it is desirable to use alternative methods which are less expensive from one hand and can secure reliable information about reservoir and hydrocarbon properties on the other hand. Among several challenges for identifying and evaluating laminated shaly sand (LSS), the following two are worth mentioning. First and foremost, the thickness of the thin beds is less than the resolution of conventional logs and consequently, conventional well logs fail to identify these layers. Second, the interpretation method for producing an output record medium which clearly illustrates each layer associated with a plurality of layers of a laminated shaly sand formation traversed by a wellbore, the output record medium quantifying each layer of the plurality of layers by providing the resistivity, or other such parameter of each layer (Passey et al. 2006).

Which method can clearly illustrate and quantify each layer of the laminated shaly sand intervals in the wellbore?

For clearly illustrating and quantifying each layer of the LSS, a technique must be developed to enhance a low-resolution measurement's vertical resolution. Several techniques have been developed to enhance a low-resolution measurement by combining it with another measurement which has a much better vertical resolution. To enhance vertical resolution of the low-resolution measurement, considerable work has been appeared in the literature. According to the results of the different techniques, the best results have been obtained when the high-resolution square log method used for studying the LSS formations. Finally, we used highresolution square logs for evaluating the thin-bed layers in this research.

The high-resolution square log method and apparatus receiving a low-resolution log and a high-resolution log from a well logging tool disposed in the wellbore and generating in response there to rectangular deconvolved log which is subsequently convolved with a vertical response function of the well tool there by producing a new reconstructed log, the new reconstructed log undergoing interactive refinement and producing a new refined rectangular deconvolved log which, after undergoing volumetric analysis, produces an output record medium which clearly illustrates and quantifies each layer of the plurality of layers of the laminated formation.

This paper is organized as follows: first, we discuss the geology of studied oil field well which covering the: location and lithology of the formation. Also the available logs (conventional logs, image $\log$ ) in the studied formation are presented in this section. The high-resolution square log method for enhancing vertical resolution of the low-resolution measurement is completely described in the next section. MATLAB-based computer program which is developed to generate square logs is presented in this section. Finally, we demonstrate application of the high-resolution square log technic for analyzing the LSS interval with field cases in the last section.

\section{Geology of studied oil field}

The onshore Ahwaz oilfield is located at the end of the folded Zagros zone near Ahwaz City in the south/southwest of north Dezful embayment. It is one of the largest hydrocarbon bearing structures in the world, and actually it is a large northwest-southwest trending anticline with a subsurface area of 80 by $6.5 \mathrm{~km}$. Currently, Iran's largest producing field is the onshore Ahwaz-Asmari field which is located in Khuzestan province. An aerial map illustrating the stratigraphy of Zagros is given in Fig. 1 (Memari 2013).

\section{Asmari Formation (Oligo-Miocene)}

Asmari Formation is located underneath anhydrite Gachsaran Formation. Gachsaran forms the caprock at many important oil fields. Evaporites in the overlying lowermost member of the Gachsaran Formation form an efficient caprock for the Asmari reservoir. The Asmari Formation (OligoMiocene) is the major oil reservoir in Iran and is mainly composed of carbonate entities (Limestone and Dolomite). The Asmari Formation produces almost 85 percent of total Iranian crude oil, and it is one of the main known reservoirs in the world. Therefore, the study of this rock unit has been a critical subject in the past and in the present time (Amani 2013) (Fig. 2).

The well analyzed in this study is a vertical well that is completed in black oil reservoir within the Asmari Formation. The geological and petrophysical studies in Asmari Formation show the existence of the laminated shaly sand intervals in the base of Asmari Formation. The LSS section of this well was drilled with an 8.5 inch bit and oil-base mud. 


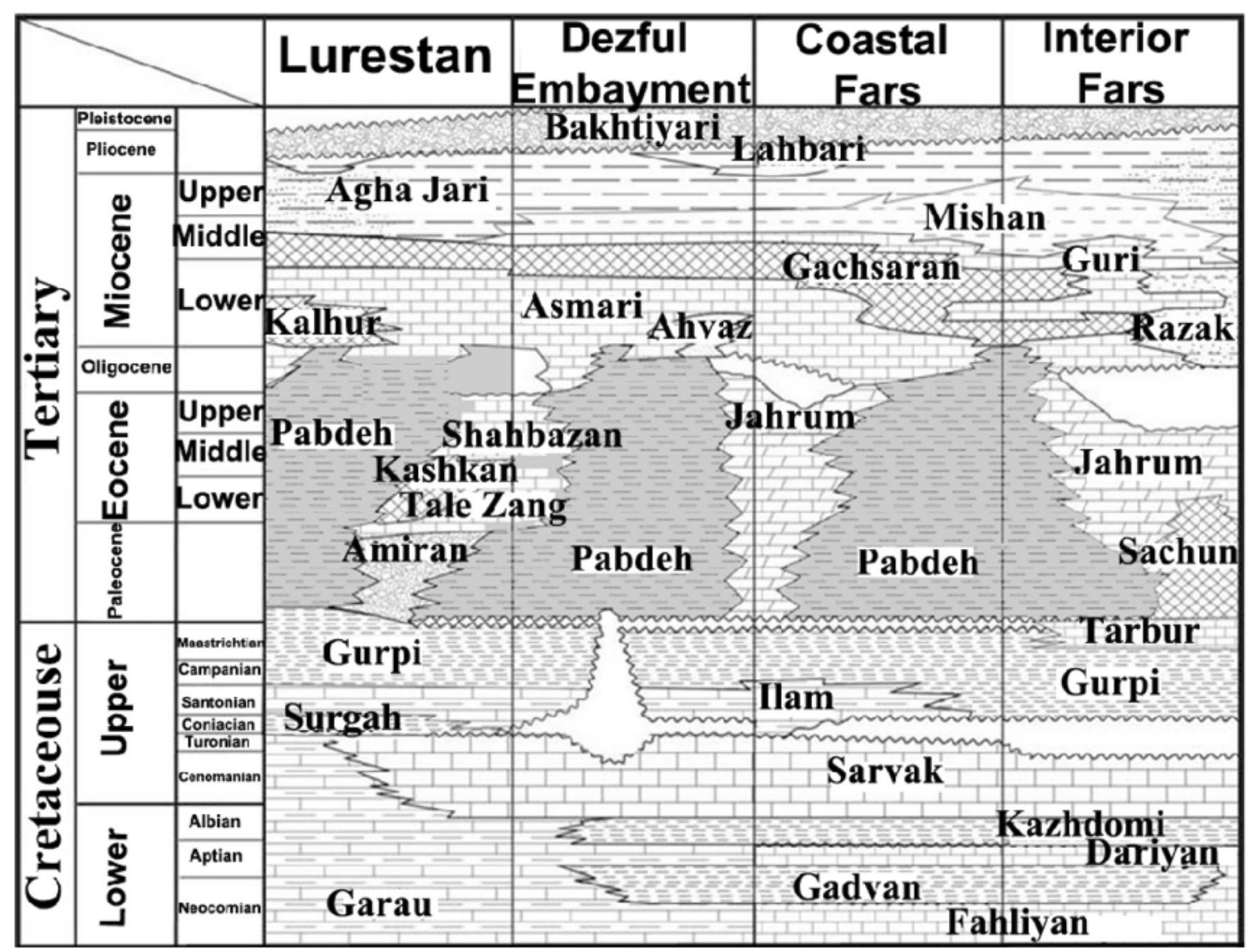

Fig. 1 Stratigraphy of Zagros (Tabatabaei 2017)

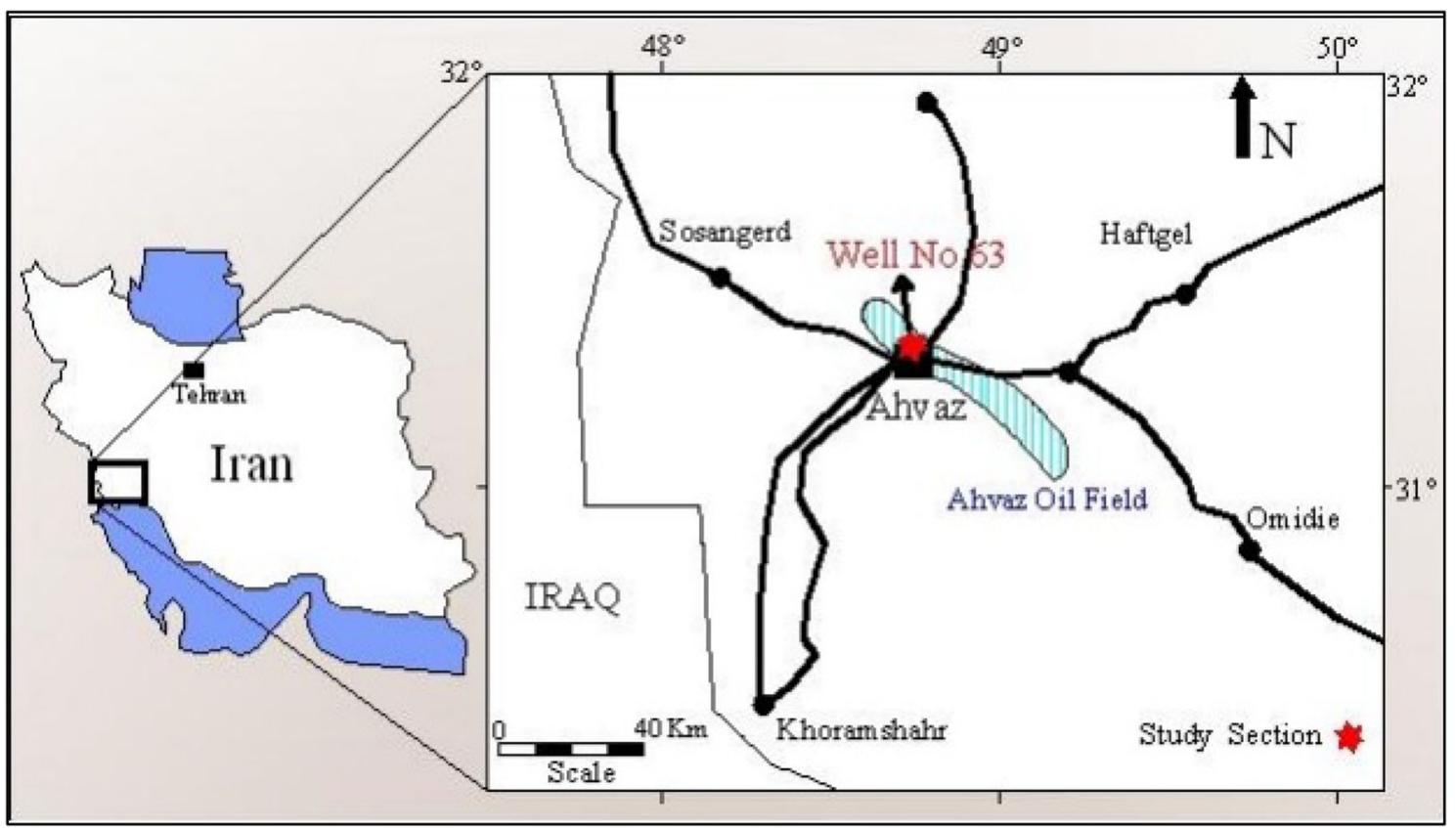

Fig. 2 Location of Ahwaz oil field in Southwest of Iran (Asghari and Adabi 2014) Ahwaz oil field contains different lithologies which one of them is Asmari Formation. The lithological description of this formation is as follows 
Measurements include a complete suite of conventional logs and resistivity image logs. Figure 3 shows the conventional and image logs were run in LSS part of this well within the studied reservoir.

\section{Description of the high-resolution square log method}

LSS parts are composed of alternating beds of low resistivity of shales and high resistivity of sand layers. The limited vertical resolution of conventional wireline tools causes conventional petrophysical analysis yields only average petrophysical properties across LSS Formations. High-resolution square log method is advantageous over the conventional $\log$ s as each individual bed can be analyzed separately which in turn yield more accurate estimation of hydrocarbon reserves (Bastia et al. 2007). The high-resolution square log approach was first described by Tabanou and Antoine (Tabanou and Antoine 1995) and includes the following steps:

\section{Bed boundary identification}

Bed boundaries can be identified by various methods. One method is from inflection points which are defined based on maximum slope change (second derivative method) in image log. The other method is based on the histogram. The histogram is a plot resistivity of the LSS formation on the $x$-axis as determined by the image log, and the $y$-axis is the frequency of occurrence. As is shown in Fig. 4, this histogram has two cutoff values (cutoff 1 and cutoff 2). According to these cut offs value, the three modes 1 , mode 2 and mode 3 have been separated from the histogram. Mode 1 includes the intervals which exists to the left of cutoff 1 area with green color, mode 2 consists of the intervals which exists between cutoff 1 and cutoff 2 areas with yellow color, and mode 3 consists of the intervals which exists to the right of cutoff 2 areas with red color. Finally, average value of the each mode was determined from the histogram (such as: $a_{1}$ average value of the mode $1, a_{2}$ average value of the mode 2 and $a_{3}$ average value of the mode 3 ).

\section{Basic square log generation}

Basic square log generation is described in this step. For this purpose, the depth is plotted versus resistivity (Fig. 5). This figure is a plot of depth on the $y$-axis and resistivity of the LSS formation on the $x$-axis as determined by the image log developed from the high-resolution well logging tool. On the $x$-axis, the cutoff values are marked then according to the cutoff values, the figure is separated to the different modes. Then each depth is attributed to one of the modes according to cutoff value location.

Resistivity value of each depth replaced with the average value of each mode which determined in the above $\left(a_{1}\right.$,

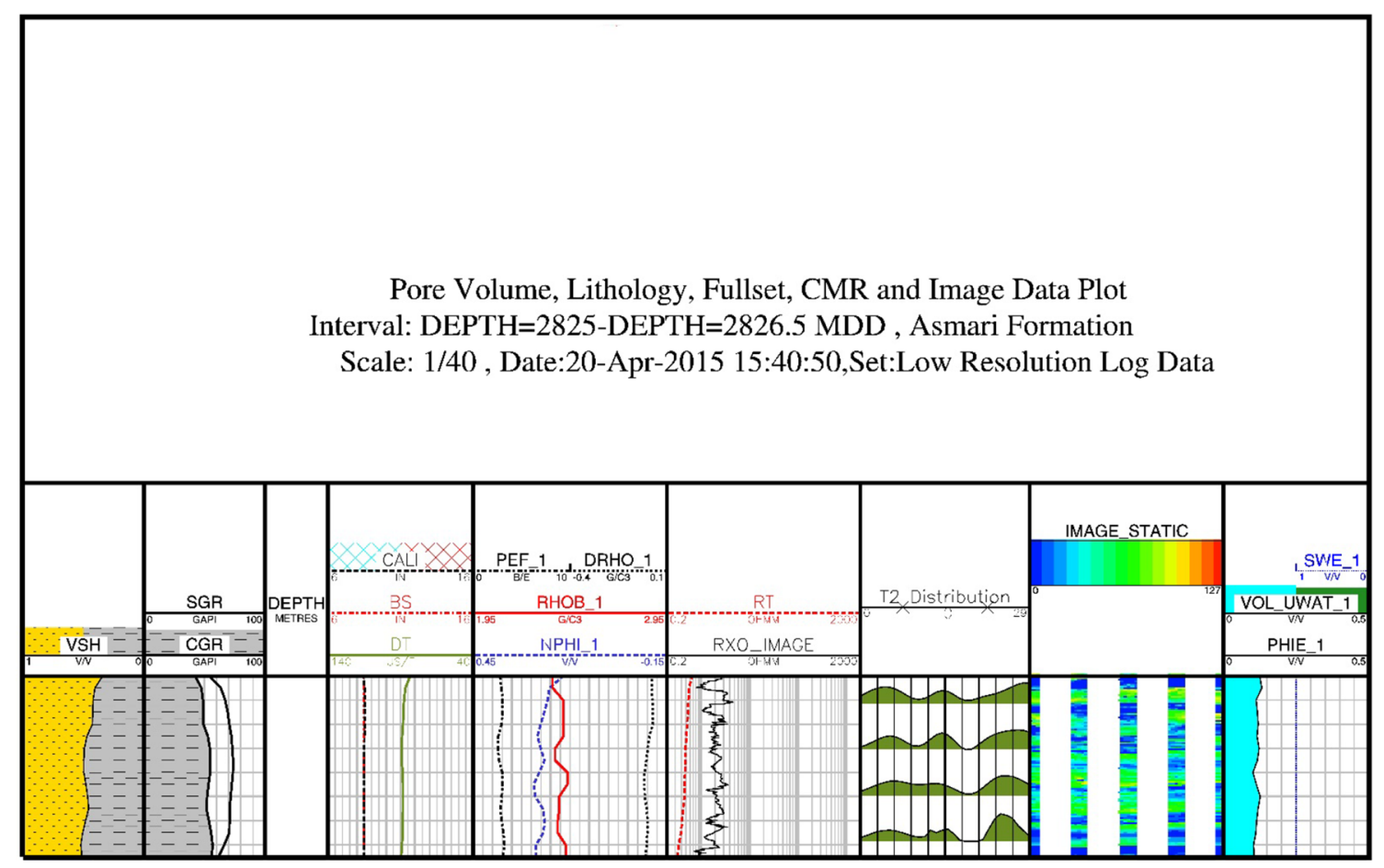

Fig. 3 Available logs in the LSS section

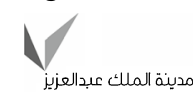

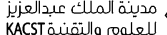
Springer 


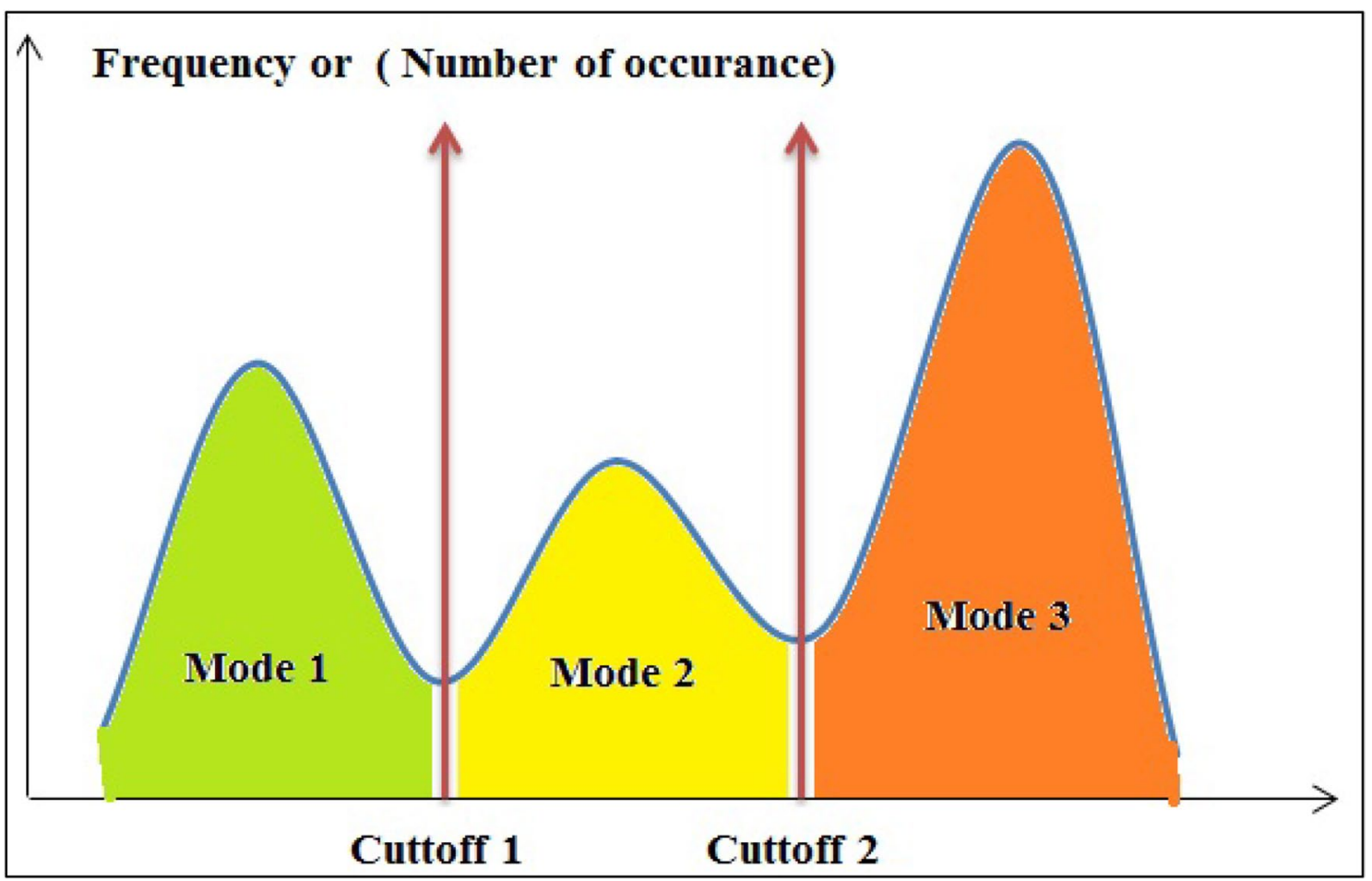

Fig. 4 Histogram of the image log data

$\mathrm{a}_{2}$ and $\mathrm{a}_{3}$ ) according to the identified mode for each depth (Fig. 6).

Finally, in Fig. 6, values are connected and the Basic square $\log$ is generated.

\section{Classification of the faces}

The finite numbers of modes are classified from the histogram of a high-resolution measurement. From a geological view also, the important principle can conclude that laminar thin-bed reservoirs are often the result of a cyclic sedimentary process which tends to deposit similar materials in cycles over long period of times. Based on the geological information and the field experience in the LSS intervals, one can conclude the LSS sequences often can be classified into two main faces: sand and shale. These faces' petrophysical properties can be identified by using conventional logs.

\section{Determining the different logs constraint}

It can be concluded from preceding that the shale and sand in the LSS sequences are the same as the thick shale and sand layers in the nearby the LSS sequences. So, maximum and minimum (or constraint) value of different logs (such as: CGR $\log$, resistivity $\log$, NPHI log and RHOB $\log$ ) of these faces could be determined from the disposed conventional $\log$ of nearby thick sand and shale layers. Table 1 shows the constraint of the different logs.

\section{Square log generation}

In this step, the value of each log of the faces which is determined in the previous step is considered as that face value and subsequently, the primary square $\log$ of each $\log$ was generated (Fig. 7).

\section{Obtain the optimized square logs}

Each log tool has a vertical response function. After generation of initial set of square $\operatorname{logs}$, $\log$ s similar to the standard resolution logs should be produced. This is called constructed $\log$. Therefore, the square log of each log type should be convolved with the vertical response function of that $\log$ (Fig. 8).

After generation of constructed logs, optimizer would check whether the constructed logs are matching with the standard $\log$ or not, if they are not matched then the square logs iteratively change between the constraints value (maximum and minimum) for each log then convolved with the response function and matched with the standard log; therefore, this process continued until the constructed $\log$ and the standard log to get a best match. Then, the final square log which the constructed $\log$ of that $\log$ is matched with the standard log selected and 


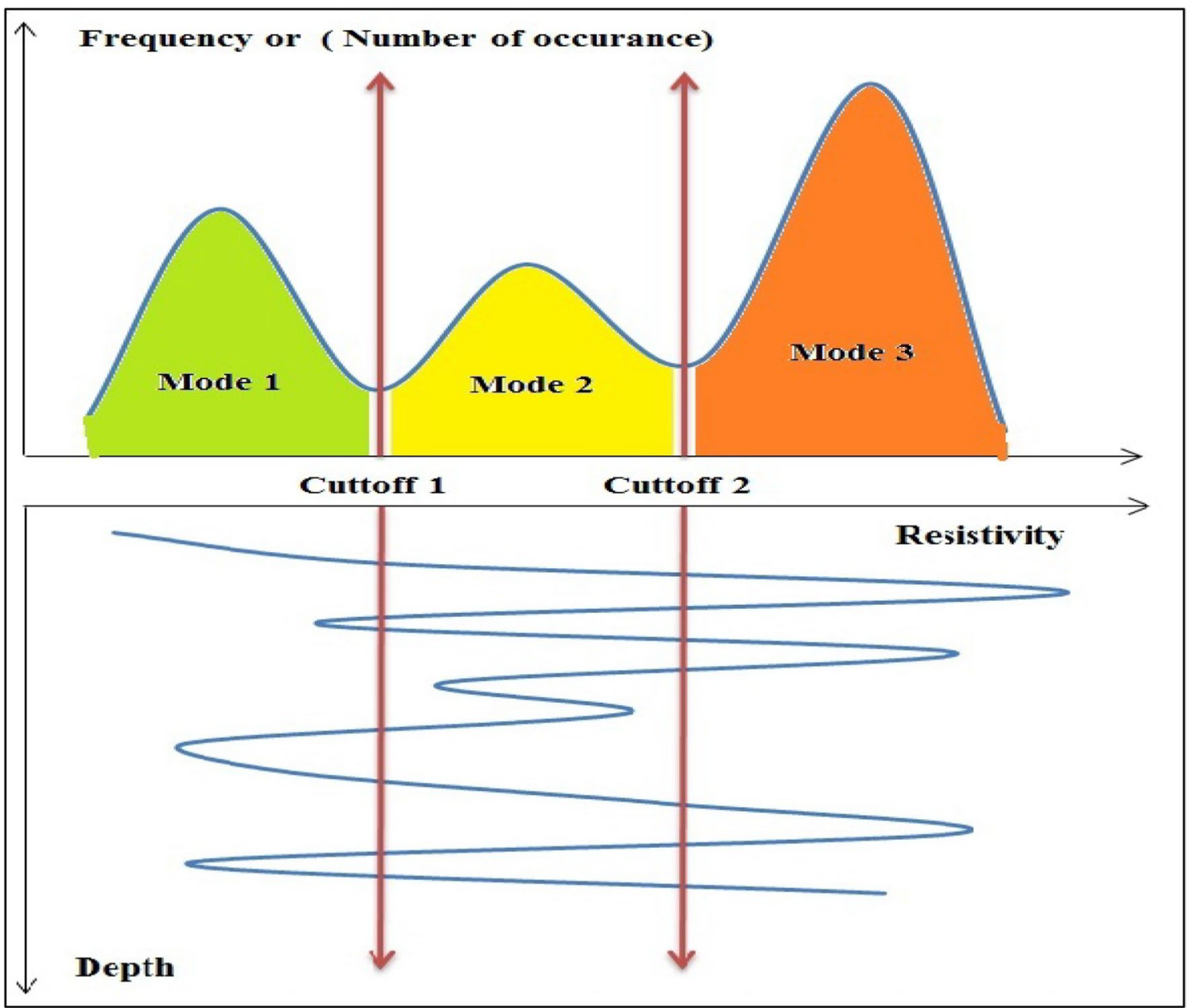

Fig. 5 Plot of the image log

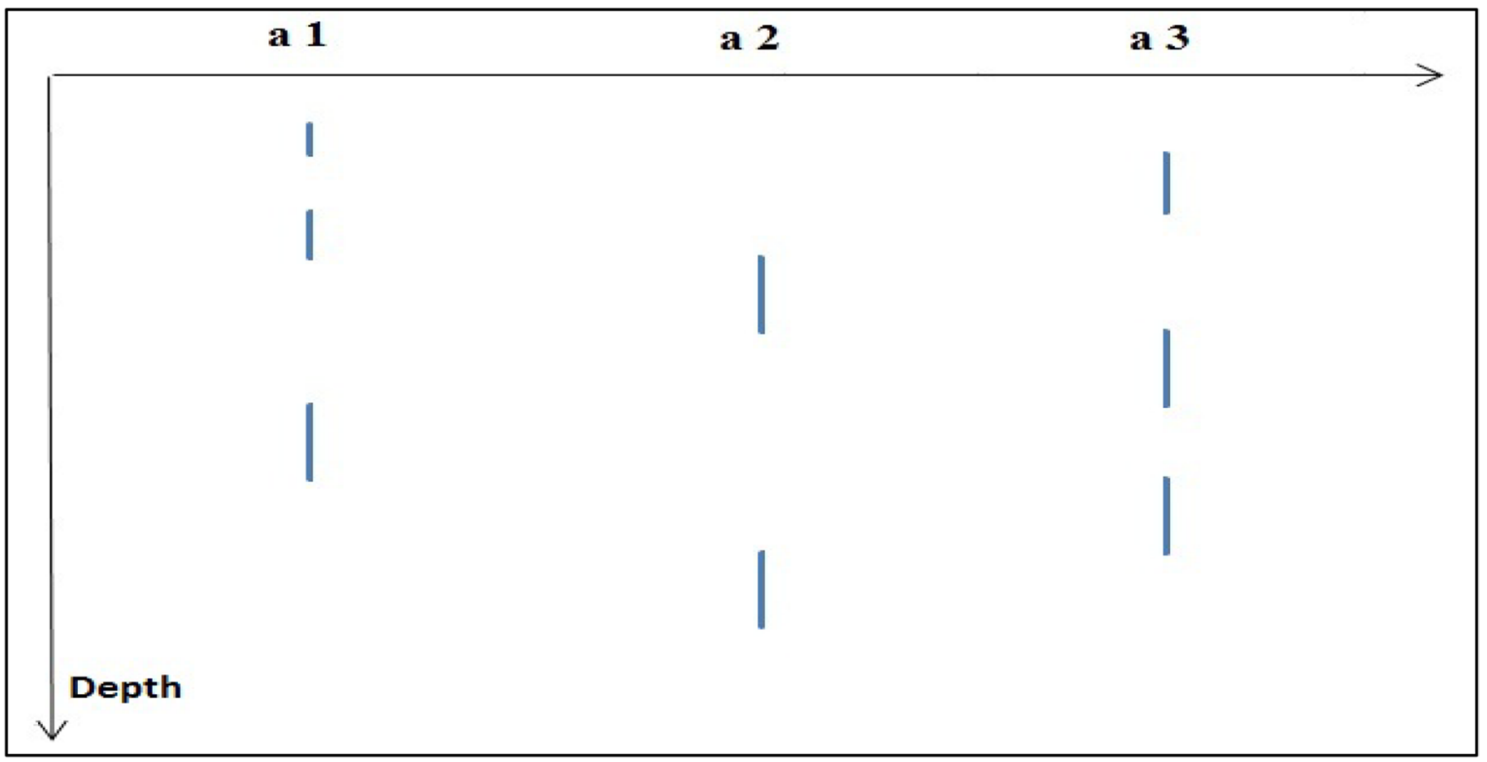

Fig. 6 Replace the average value with the real value 
Table 1 Different logs constraint

\begin{tabular}{|c|c|c|}
\hline Log type & Maximum value & Minimum value \\
\hline CGR & $\begin{array}{l}\text { Disposed conventional CGR log value of the nearby thick shale } \\
\text { layer }\end{array}$ & $\begin{array}{l}\text { Disposed conventional CGR log value of the nearby thick sand } \\
\text { layer }\end{array}$ \\
\hline RHOB & $\begin{array}{l}\text { Disposed conventional RHOB log value of the nearby thick } \\
\text { shale layer }\end{array}$ & $\begin{array}{l}\text { Disposed conventional RHOB log value of the nearby thick sand } \\
\text { layer }\end{array}$ \\
\hline NPHI & $\begin{array}{l}\text { Disposed conventional NPHI log value of the nearby thick shale } \\
\text { layer }\end{array}$ & $\begin{array}{l}\text { Disposed conventional NPHI log value of the nearby thick sand } \\
\text { layer }\end{array}$ \\
\hline Resistivity & $\begin{array}{l}\text { Greater value of the disposed conventional resistivity log value } \\
\text { of the nearby thick shale and sand layer }\end{array}$ & $\begin{array}{l}\text { Lower value of the disposed conventional resistivity log value of } \\
\text { the nearby thick shale and sand layer }\end{array}$ \\
\hline
\end{tabular}

The value of resistivity log of the sand and shale layers in the LSS is depended to the filled fluid saturation of those layers. Resistivity of the shale and sand according to their saturation can be maximum or minimum. The resistivity log of the thick shale and sand layer of the near LSS sequences is read from disposed conventional $\log$ and then the greater value is put as maximum and the lower value is put as minimum constraints of the resistivity $\log$

For obtaining the final reliable results with less iterations, these constrains should be identified

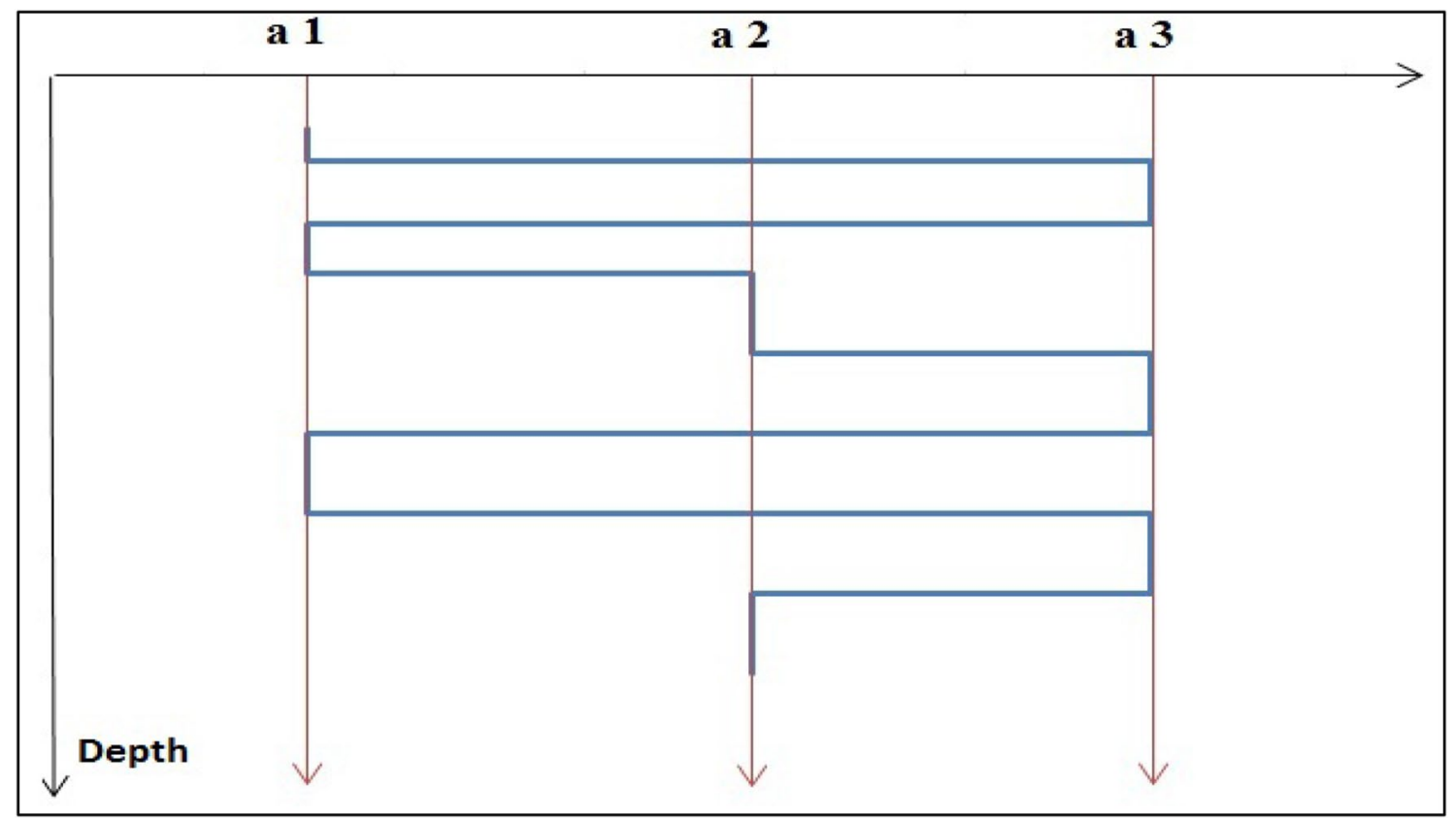

Fig. 7 Basic square log

named as optimum square log (Fig. 9). These optimum square logs are the best high-resolution square log which can exactly evaluate the LSS formation. Finally, the volumetric petrophysical analysis is done on the optimum square logs.

The flowchart for the high-resolution approach is shown in Fig. 10.

\section{Square log generation program}

So far, the generations of high-resolution square logs were described. Generation of square $\log$ is an iterative and time consuming method, which requires very advanced mathematical calculations. A MATLAB-based (MATLAB 2014) computer program is developed to generate accurate square logs according to the described method. The developed code can perform all the steps efficiently in reasonable timeframe. In this software, low-resolution logs and high-resolution (Image) log, different logs constraints and number of histogram bins are given as input. Then, square logs are calculated and plotted versus low-resolution logs. This program could help petrophysics engineering to analyze the LSS effectively. A graphical user interface (GUI) built to perform interactive tasks is illustrated in Fig. 11. 


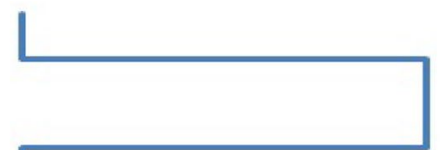

\section{Tool Response Function}
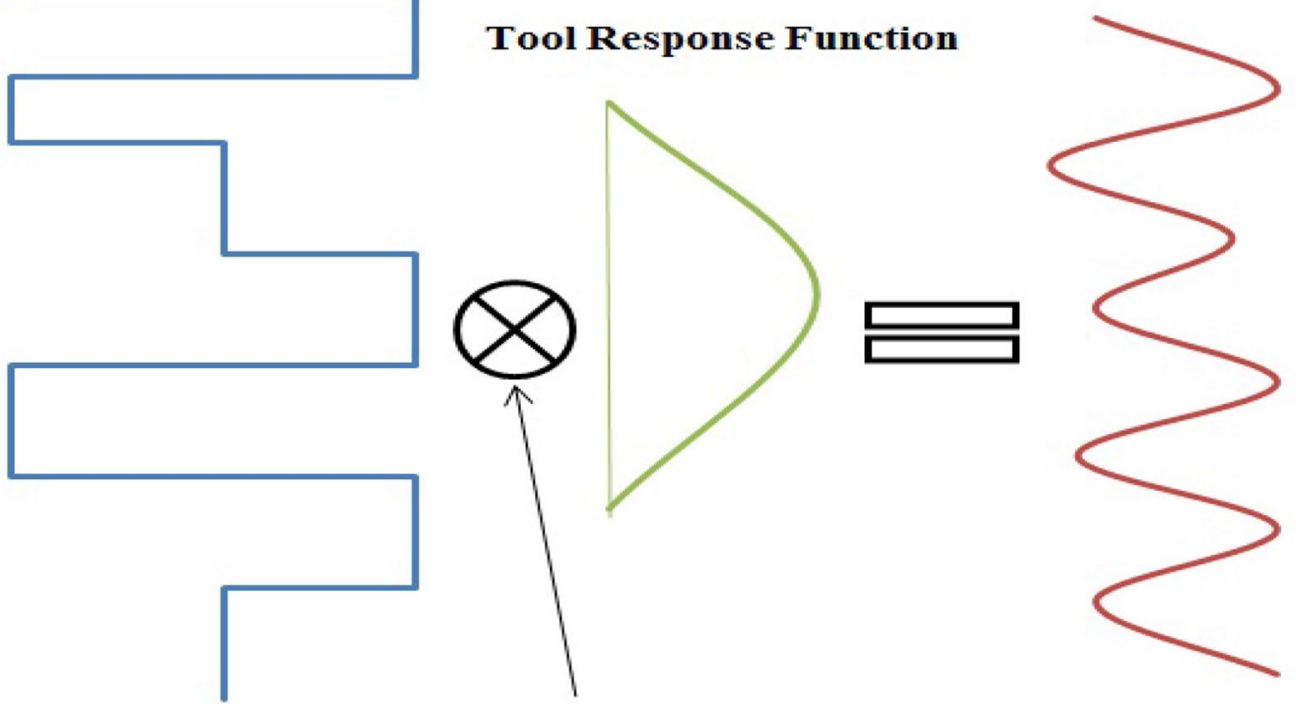

Square log

Convolution

Constructed log

Fig. 8 Obtaining the constructed log from square log

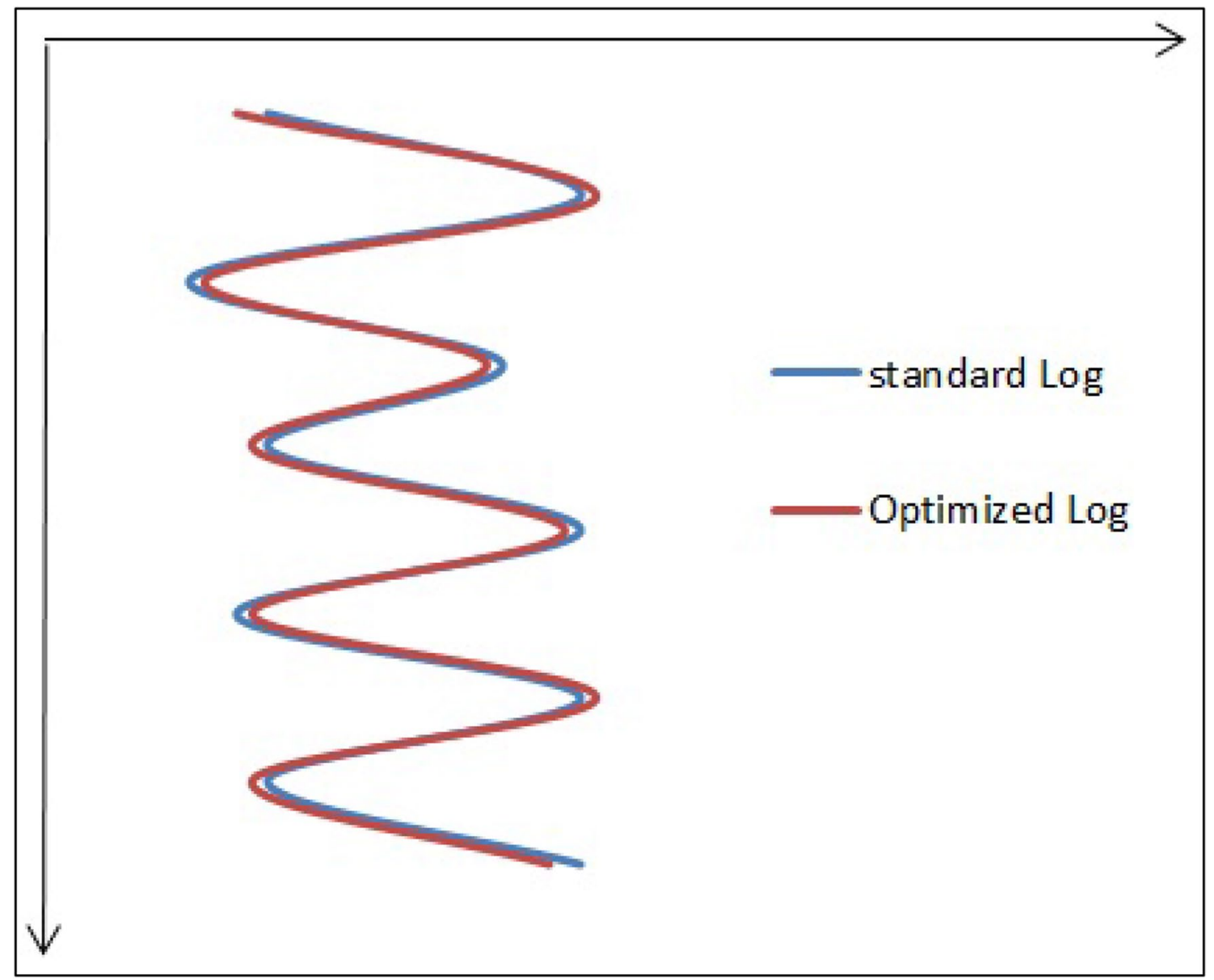

Fig. 9 Matching the constructed log with the standard log 


\section{Field example}

This field case examines thinly bedded sand-shale sequences encountered in Well A. Negligible invasion effect was observed in apparent resistivity curves with different radial lengths of investigation, which indicates the absence of mobile water pore volume in the formation. In this paper, a thin-bed analysis was also performed over the LSS section with two methods: convectional and square logs. These logs were analyzed with deterministic method by using the Geolog 7.2 software. Figures 12 and 13 show the logs and results of petrophysical analysis of these logs.

A good agreement was found between the laminae identified on the square logs and the laminae from image $\log$. This result adds confidence to the calculated properties with this method (Figs. 14 and 15).

\section{Petrophysical parameter calculation from conventional and square logs methods}

Table 2 compares the calculated petrophysical parameters (i.e., water saturation, porosity and net hydrocarbon column) from conventional and square log methods. The estimated net hydrocarbon column in LSS is considerably enhanced using the square logs results compared with using conventional $\log$ s results.

From the results of Table 3 , it is concluded that conventional petrophysical analysis ignored net hydrocarbon column in laminated sands when compared to those calculated with square $\log$ s analysis. Also, water saturation estimated with square $\operatorname{logs}$ analysis is about 43.6 percent which is lower than the NISOC cutoffs. Porosity estimated with square $\log$ s analysis is about 24.5 percent which is upper than the NISOC cutoffs.

We concluded that conventional petrophysical analysis ignored net hydrocarbon column in laminated sands when
Fig. 10 Workflow diagram for thin-bed analysis with highresolution approach

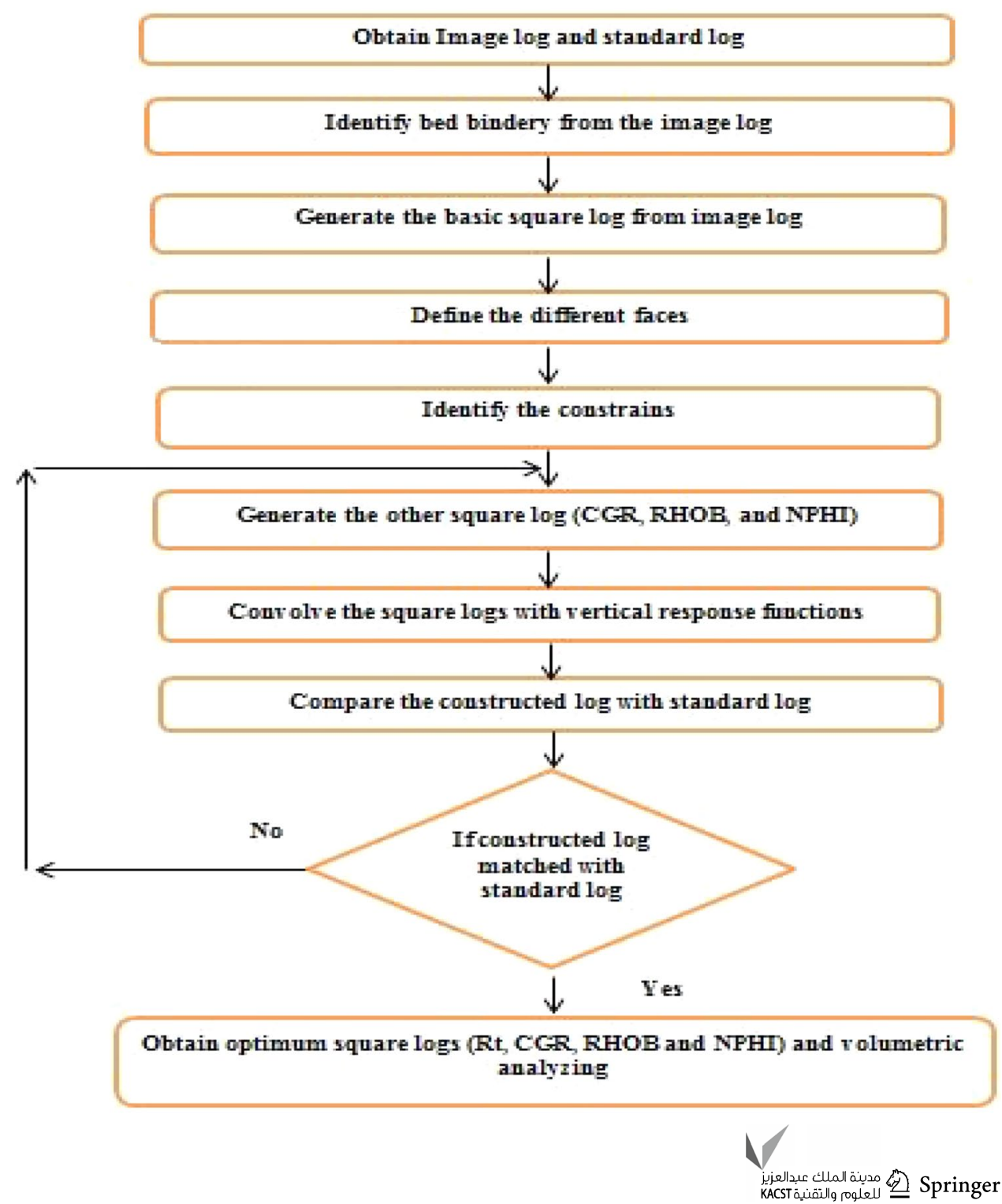




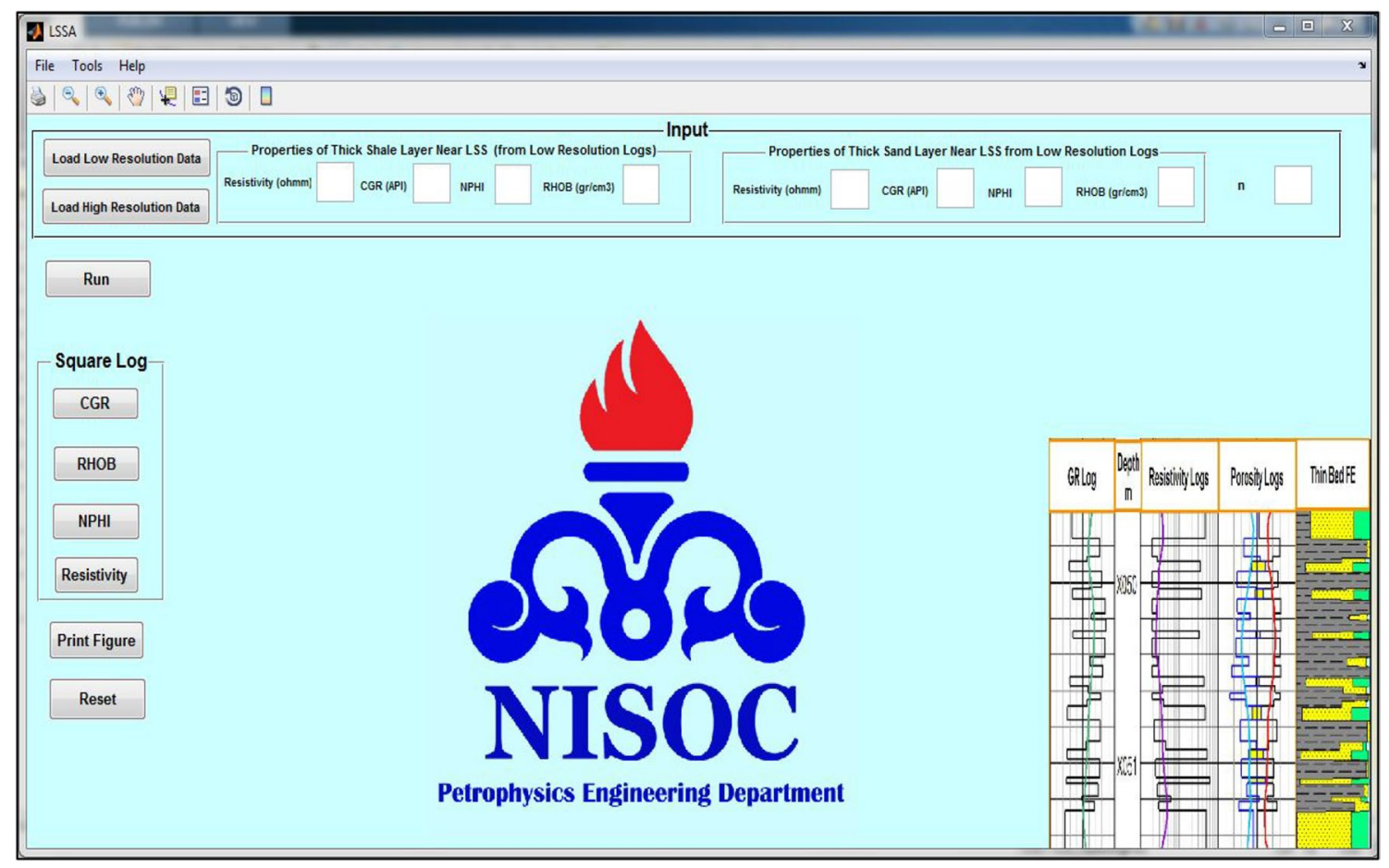

Fig. 11 Software graphical user interface for square log generation program

Pore Volume, Lithology, Fullset, CMR and Image Data Plot Interval: DEPTH=2825-DEPTH=2826.5 MDD , Asmari Formation

Scale: 1/40 , Date:20-Apr-2015 15:40:50,Set:Low Resolution Log Data

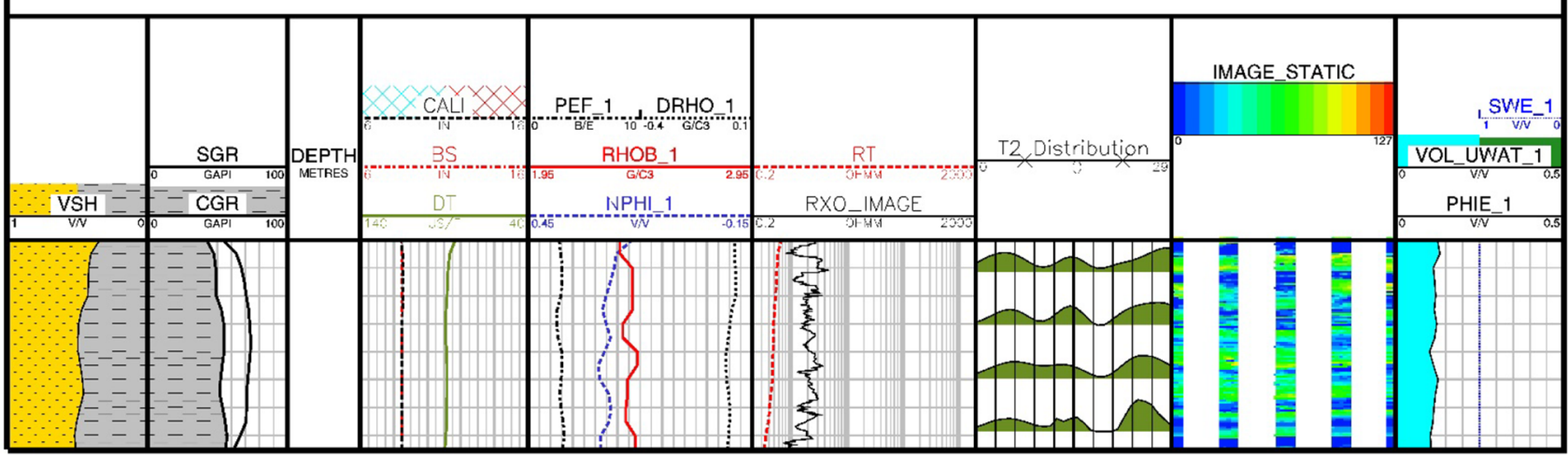

Fig. 12 Conventional logs results 
Pore Volume, Lithology, Fullset, CMR and Image Data Plot Interval: DEPTH=2825-DEPTH=2826.5 MDD , Asmari Formation

Scale: 1/40, Date:20-Apr-2015 15:40:50,Set:Square Log Data

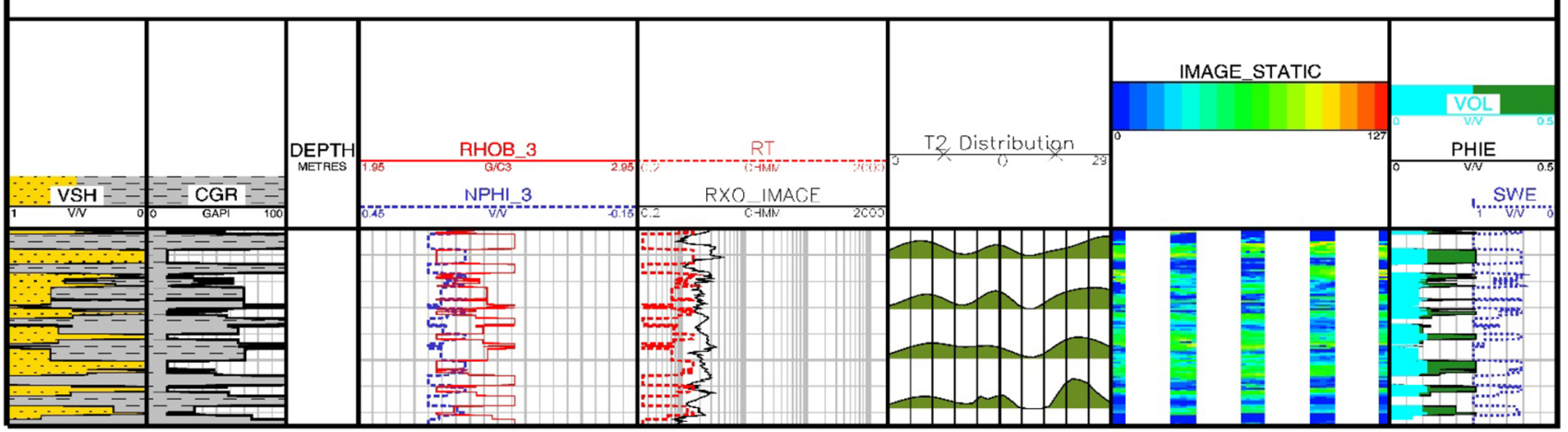

Fig. 13 Square logs results

compared to those calculated with square logs analysis. Also, water saturation estimated with square logs analysis is about 56.6 percent which is lower than the conventional $\log$ analysis. Porosity estimated with square logs analysis is about 11.3 percent which is lower than the conventional $\operatorname{logs}$ analysis.

\section{Conclusions}

In this paper, square logs have been used for improving the formation evaluation of low-resistivity pay reservoirs in LSS sequences. Both of the high- and low-resolution $\log$ s are used in generating the square $\log$. This process yields a set of high-resolution square $\log$ s that can be lead to more accurate estimations of petrophysical parameters. Square $\log$ s helping to reduce errors and reserve
Fig. 14 Petrophysical parameter with consideration of the NISOC cutoffs

\section{Petrophysical parameter with considering the NISOC cutoffs}

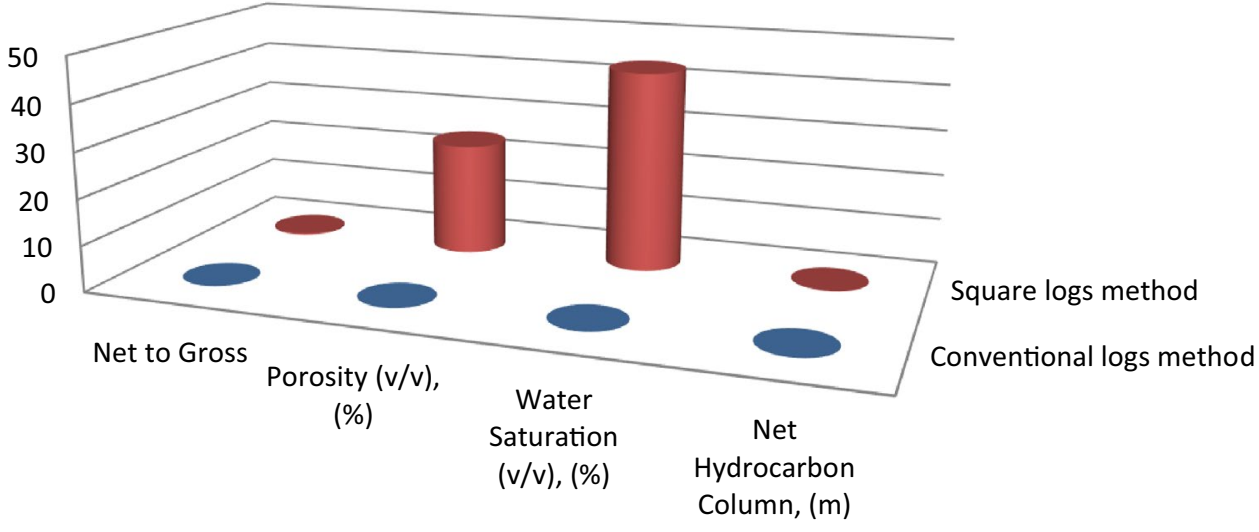


Fig. 15 Petrophysical parameter without consideration of the NISOC cutoffs

\section{Calculated petrophysical parameter without NISOC cutoffs}

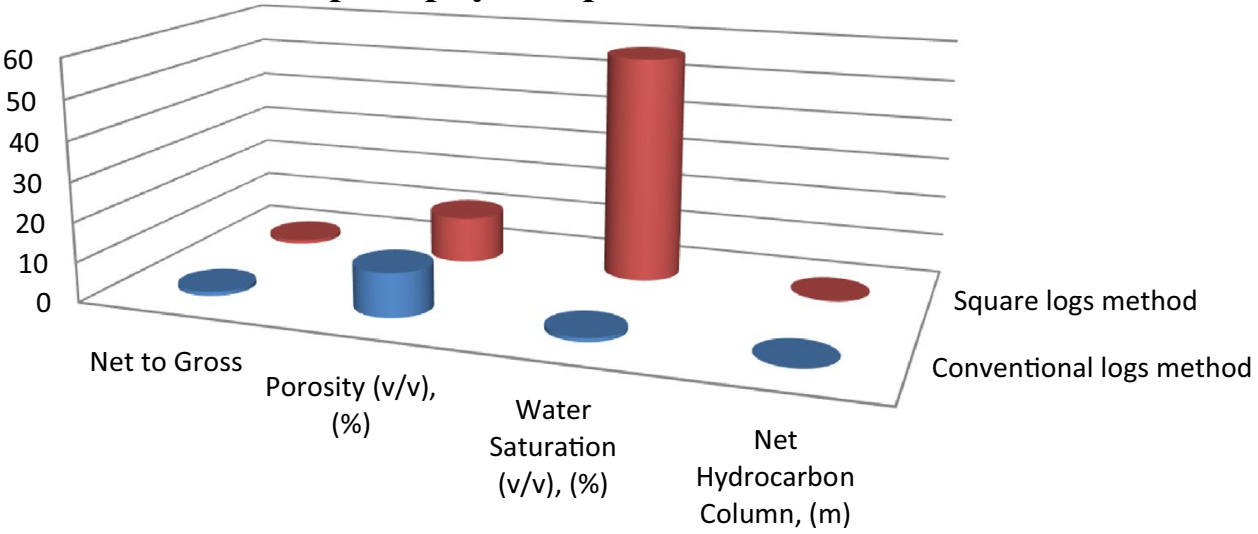

Table 2 Petrophysical parameter calculated from conventional (the standard resolution) and square (high resolution) logs methods. NISOC cutoffs used: effective porosity $>=0.045$, effective water saturation $<=0.5$, volume of clay $<=0.5$

\begin{tabular}{lll}
\hline & $\begin{array}{l}\text { Conventional logs } \\
\text { method }\end{array}$ & $\begin{array}{l}\text { Square } \\
\text { logs } \\
\text { method }\end{array}$ \\
\hline Net to gross & 0 & 0.276 \\
Porosity (v/v), (\%) & 0 & 24.5 \\
Water saturation (v/v), (\%) & 0 & 43.6 \\
Net hydrocarbon column, $(\mathrm{m})$ & 0 & 0.1 \\
\hline
\end{tabular}

Table 3 Petrophysical parameter calculated from conventional (the standard resolution) and square (high resolution) logs methods without cutoffs

\begin{tabular}{lll}
\hline & $\begin{array}{l}\text { Conventional logs } \\
\text { method }\end{array}$ & $\begin{array}{l}\text { Square } \\
\text { logs } \\
\text { method }\end{array}$ \\
\hline Net to gross & 1 & 1 \\
Porosity (v/v), (\%) & 11.1 & 11.7 \\
Water saturation (v/v), (\%) & 1 & 56.6 \\
Net hydrocarbon column, (m) & 0 & 0.073 \\
\hline
\end{tabular}

uncertainty, especially in cases where $\mathrm{Rv}$ and $\mathrm{Rh}$ data are not available.

The square logs method was used for thin-bed petrophysical evaluation in a well, North West of Iran. The main conclusions obtained from the square logs petrophysical evaluation in this well are as follows:

1. Thin beds were fairly well identified by using the highresolution square log technique.

2. Thin beds, contained significant amounts of the total hydrocarbon reserves.
3. The square logs petrophysical evaluation results were compared to the conventional petrophysical evaluation in two aspects:

a. Using the same NISOC cutoffs for both square and conventional logs method, net pay calculations, yielded net hydrocarbon column of $0.1 \mathrm{~m}$ and $0 \mathrm{~m}$, porosity of $24.5(\%)$ and $0(\%)$, water saturation of $43.6(\%)$ and $0(\%)$.

b. Using the without cutoffs for both square and conventional logs methods net pay calculations, yielded net hydrocarbon column of $0.044 \mathrm{~m}$ and $0 \mathrm{~m}$, porosity of $11.7(\%)$ and $11.1(\%)$, respectively, i.e., a $5.4 \%$ increase approximately and also water saturation of $56.6(\%)$ and 1 (\%),respectively, i.e., a $43.4 \%$ decrease approximately.

4. Developed computer program for generating square $\log$ s is very accurate and is very useful in thinly bedded sequences analysis.

Acknowledgements The authors appreciate the petrophysics engineering division of (NISOC) for providing petrophysics and geological data presented in this work.

Open Access This article is distributed under the terms of the Creative Commons Attribution 4.0 International License (http://creativeco mmons.org/licenses/by/4.0/), which permits unrestricted use, distribution, and reproduction in any medium, provided you give appropriate credit to the original author(s) and the source, provide a link to the Creative Commons license, and indicate if changes were made.

\section{References}

Amani A (2013) Prediction of uniaxial compressive strength using drilling data and sonic logs to recommend the best bit type for iranian south oil formations. M.Sc. theses, Petroleum University of Technology

Asghari M, Adabi MH (2014) Digenesis and geochemistry of the sarvak formation in ahvaz oil field-Iran. Geochem J 1:2 
Azim SA, Abdullah SM, Al-Zaabi H, Al-Awadi.W, Najeh H, Bryant WB, Aldred R, Steel M (2005) Resistivity anisotropy and its impact on reserves estimates and technology applications in North Kuwait reservoirs. In: SPE 93503, presentation at the 14th SPE middle east oil \& gas show and conference held in Bahrain International Exhibition Centre, Bahrain, 12-15 Marchpp, 2-4

Bastia R, Tyagi A, Saxena K, Reliance Industries Ltd., Klimentos T, Altman R, Alderman S, Bahuguna S (2007) Evaluation of lowresistivity-pay deep water turbidities using constrained thin-bed petrophysical analysis. In: SPE 110752, presentation at the 2007 SPE annual technical conference and exhibition held in Anaheim, California, U.S.A., November, 11-14

Coates GR, Xiao L, Prammer MG (1999) NMR logging principles and applications. Halliburton Energy Services Publication, Houston

Gabriela AM, Lorne AD (2000) Petrophysical measurements on shales using NMR. In: SPE/AAPG Western Regional Meeting, Long Beach, California

Memari A (2013) Evaluation of surface subsidence in one of Iran's oil fields using INSAR1 technique. Am J Oil Chem Technol 1(4):9-17

Passey QR, Dahlberg KE, Sullivan K, Yin H, Brackett B, Xiao Y, Guzman-Garcia AG (2006) Petrophysical evaluation of hydrocarbon pore-thickness in thinly bedded clastic reservoirs: AAPG Archie Series, No. 1, Tulsa
Tabanou J, Antoine J (1995) Method and apparatus for detecting and quantifying hydrocarbon bearing laminated reservoirs on a workstation. United States Patent 5461562, Schlumberger Technology Corporation and Houston, Tex. Oct. 24

Tabatabaei H (2017) Potential evaluation and basin modeling of the pabdeh formation in zagros basin: a case study. Open J Geol 7:505-516

Vielma M, Vandini R (2007) Hydrocarbon reserves and production increment in laminated sand/shale reservoir from $3 \mathrm{~d}$ resistivity measurements, image logs, and Magnetic Resonance Technology. In: Latin American \& Caribbean Petroleum Engineering Conference, SPE 107689, Argentina, 15-18 April, pp. 1-9

Zhang Z et al. (2007) Triaxial induction logging —an operations perspective. In: Society of petrophysicists and well log analysts (SPWLA) 48th annual logging symposium held in Austin, Texas, United States, June 3-6, 2-8

Publisher's Note Springer Nature remains neutral with regard to jurisdictional claims in published maps and institutional affiliations. 